\title{
Activation of protease-activated receptor-2 is associated with increased expression of inflammatory factors in the adipose tissues of obese mice
}

\author{
MENGMENG LI ${ }^{1}$, XIAOMING YANG ${ }^{2}$, YADONG ZHANG $^{3}$, LIANG CHEN $^{1}$, \\ HANYU LU ${ }^{1}$, XIAOBO LI ${ }^{1}$, LIANHUA YIN ${ }^{1}$ and XIULING ZHI ${ }^{3}$ \\ ${ }^{1}$ Department of Physiology and Pathophysiology, School of Basic Medical Sciences, Fudan University, Shanghai 200032; \\ ${ }^{2}$ Department of Pathophysiology, School of Basic Medical Sciences, Ningxia Medical University, Yinchuan, \\ Ningxia 750004; ${ }^{3}$ Laboratory of Molecular Biology, Training Center of Medical Experiments, \\ School of Basic Medical Sciences, Fudan University, Shanghai 200032, P.R. China
}

Received October 10, 2014; Accepted July 3, 2015

DOI: $10.3892 / \mathrm{mmr} .2015 .4179$

\begin{abstract}
Previous studies have indicated that mast cells are critical for the pathogenesis of inflammatory diseases. Proteases released from mast cells have been reported to stimulate protease-activated receptors (PAR), which induces microleakage and widespread inflammation. In order to investigate the pro-inflammatory effect of PAR-2 activation on adipose inflammation in obese mice, the varying distributions of macrophages and PAR-2 in adipose tissue samples were compared between $\mathrm{C}_{57} \mathrm{BL} / 6 \mathrm{~J}\left(\mathrm{C}_{57}\right)$ and obese mice [B6(D)-Lepr $r^{\mathrm{db}} / \mathrm{J}, \mathrm{BKS}(\mathrm{D})-L e p r^{\mathrm{db} / \mathrm{J}}$ and B6.V-Lep $\left.{ }^{\mathrm{ob}} / \mathrm{J}(\mathrm{ob} / \mathrm{ob})\right]$ using immunohistochemical staining. Murine primary adipocytes and bone marrow-derived macrophages (BMDMs) were used and the alterations in expression levels of inflammatory factors, induced by PAR-2 activation, were detected by reverse transcription-quantitative polymerase chain reaction and ELISA. In addition, the migratory capacity of primary BMDMs and the macrophage cell line, RAW264.7 were evaluated by co-culture with primary adipocytes. The current study demonstrated a larger number of macrophages in the adipose tissues of obese mice compared with $\mathrm{C}_{57}$ mice. Furthermore, PAR-2 expression was detected in various adipose tissues of mice and the protein expression levels of PAR-2 were observed
\end{abstract}

Correspondence to: Professor Lianhua Yin, Department of Physiology and Pathophysiology, School of Basic Medical Sciences, Fudan University, 131 Dongan Road, Shanghai 200032, P.R. China E-mail:1hyin@shmu.edu.cn

Dr Xiuling Zhi, Laboratory of Molecular Biology, Training Center of Medical Experiments, School of Basic Medical Sciences, Fudan University, 131 Dongan Road, Shanghai 200032, P.R. China

E-mail: zhixiuling@fudan.edu.cn

Key words: protease-activated receptor-2, macrophages, adipose inflammation, obesity, mast cell to be significantly higher in the total adipose tissues of ob/ob mice when compared with the $\mathrm{C}_{57}$ mice. The expression levels of inflammatory factors were increased in adipocytes and macrophages, and enhanced migratory ability was observed in macrophages pretreated with PAR-2 agonists. The data of the current study suggests that PAR-2 is involved in the process of obesity-associated chronic low-grade systemic inflammation, which indicates that the PAR-2 signaling pathway may be a potential target for the treatment of obesity and its associated diseases.

\section{Introduction}

Chronic low-grade inflammation is a fundamental characteristic of obesity (1) and the predominant cause of metabolic disorders (2). In obese adipose tissues, increased expression levels of markers of inflammation, including interleukin (IL)-6, tumor necrosis factor- $\alpha$ (TNF- $\alpha$ ), intercellular adhesion molecule 1 and monocyte chemotactic protein-1 (MCP-1) are observed, in addition to increased infiltration of macrophages (3). Cottam et al (4) observed that inflammatory factors, such as IL- 6 and TNF- $\alpha$ in the adipose tissues were predominantly secreted by the infiltrated macrophages.

Altintas et al (5) observed that the adipose mast cells in obese mice were significantly increased and that the mast cells of the epididymal fat released IL-6 via degranulation. In a previous study, an increase in mast cells was identified in the adipose tissue of obese mice and tryptase was observed to be positive-stained in the mast cells (6). However, little is known regarding the association between mast cells and macrophages during the inflammatory process of obesity. During the degranulation process, tryptase, a key inflammatory mediator that is released from mast cells, and protease activated receptor 2 (PAR-2) activation (mediated by tryptase) may be involved in the inflammatory process of obesity (7).

PAR-2 is a G protein-coupled receptor that is widely expressed in various human cell types, which mediates multiple transmembrane signal transduction pathways, such as extracellular signal-related kinase $1 / 2$, p38 and c-Jun 
N-terminal kinase (8). PAR-2 additionally serves important physiopathological roles in tissue regeneration, angiogenesis and inflammation (8). Mast cell tryptase, trypsin and tissue factor are the predominant ligands of PAR-2 (9). Previously, the inflammatory role and associated mechanisms of PAR-2 have caused concern; previous studies have identified that activation of PAR-2 may upregulate the expression of inflammatory factors, including IL-1 $\beta$, IL- 6 and TNF- $\alpha$ in endothelial cells (10-12).

In the present study, the expression level of PAR-2 in the ob/ob obese mice was assessed. The effects of PAR-2 activation on expression of inflammatory factors were evaluated in the primary murine adipocytes, primary murine bone marrow-derived macrophages (BMDMs), and the murine macrophage cell line, RAW264.7. The current study aimed to further elucidate the pro-inflammatory role of PAR-2 in the adipose tissues of obese mice.

\section{Materials and methods}

Experimental animals and cell lines. A total of 36 male 6 -week old $\mathrm{C}_{57} \mathrm{BL} / 6 \mathrm{~J}\left(\mathrm{C}_{57}\right)$ mice were purchased from the Shanghai Laboratory Animal Center, Chinese Academy of Sciences (Shanghai, China). The B6(D)-Lepr ${ }^{\mathrm{db}} / \mathrm{J}$ (B6-db/db), BKS(D)-Lepr $r^{\mathrm{db}} / \mathrm{J}(\mathrm{BKS}-\mathrm{db} / \mathrm{db})$ and B6.V-Lep ${ }^{\mathrm{ob}} / \mathrm{J}(\mathrm{ob} / \mathrm{ob})$ mice were purchased from the Biomedical Research Institute of Nanjing University (Nanjing, China). All mice were raised in a non-sterile environment and normal light cycle (12 h/12 h) at room temperature $\left(25 \pm 1^{\circ} \mathrm{C}\right)$ and $55 \pm 5 \%$ humidity with ad libitum access to normal chow. The mice were all housed in groups of three to five individuals in plastic mouse cages $(16 \times 12 \times 28 \mathrm{~cm})$ and sacrificed within 2 weeks of purchase. Approval for the current study was obtained from the Experimental Animal Ethics Committee of Shanghai Medical College, Fudan University (Shanghai, China; permit no. 20110307-027). The RAW264.7 and L929 murine macrophage cell lines were purchased from the Type Culture Collection of the Chinese Academy of Sciences (Shanghai, China).

Immunohistochemistry. Immunohistochemistry was performed using multiple adipose tissue samples obtained from the mice in order to assess the distribution of the macrophages, including the subcutaneous, epididymal, scapular, perirenal and omental adipose tissues. The adipose tissue samples were sliced into sections of $\sim 1.5 \times 1.5 \times 0.3 \mathrm{~cm}$, then fixed in $10 \%$ formalin (Shanghai Zhenxing No. 1 Chemical Plant, Shanghai, China), gradient dehydrated, cleared and paraffin-embedded (Leica Biosystems, Wetzlar, Germany). Sections were cut and stained by immunohistochemical staining using polyclonal rabbit antibodies against F4/80 (cat. no. ab96641; Abcam, Cambridge, $\mathrm{UK}$ ) in order to identify macrophages in the adipose tissues. In brief, the slides were incubated with the rabbit anti-F4/80 polyclonal antibody $\left(1: 200\right.$ dilution) at $37^{\circ} \mathrm{C}$ for $2 \mathrm{~h}$, where normal rabbit serum (Gibco-BRL, Gaithersburg, MD, USA) served as a negative control, followed by incubation with a horseradish peroxidase-conjugated goat anti-rabbit secondary antibody (Santa Cruz Biotechnology, Inc., Santa Cruz, CA, USA; cat. no. sc-2054; $1: 100)$ at $37^{\circ} \mathrm{C}$ for $1 \mathrm{~h}$. The signals were detected using the Diaminobenzidine Substrate kit (Vector Laboratories, Inc., Burlingame, CA, USA). Counterstaining was performed with hematoxylin (Dako, Glostrup, Denmark). Fluorescent staining of the BMDMs on glass coverslips was performed using polyclonal rabbit anti-mouse F4/80 antibody (1:200 dilution; cat. no. ab111101; Abcam) and the secondary antibody, goat anti-rabbit IgG-fluorescein isothiocyanate (1:200 dilution; cat. no. sc-2012; Santa Cruz Biotechnology, Inc.). Subsequently, the cells were stained with $1 \mu \mathrm{g} / \mathrm{ml}$ DAPI (Invitrogen Life Technologies, Carlsbad, CA, USA) for $5 \mathrm{~min}$.

Primary culture of the murine adipocytes. The 6-8 week old male $\mathrm{C}_{57}$ mice were selected. Subsequent to anesthesia via an abdominal injection of $6 \%$ chloral hydrate (Sigma-Aldrich, St. Louis, MO, USA) at $0.5 \mathrm{ml} / 100 \mathrm{~g}$ of body weight and immersion disinfection (75\% ethanol, Shanghai Zhenxing No. 1 Chemical Plant), the mice were transferred to the surgery platform for sample collection. Subsequent to obtaining the samples, the epididymal adipose tissues were immediately placed into cold phosphate-buffered saline (PBS) containing penicillin $(100 \mathrm{U} / \mathrm{ml})$ and streptomycin $(100 \mu \mathrm{g} / \mathrm{ml})$ all purchased from Life Technologies (Grand Island, NY, USA). The tissues were sliced as small as possible $\left(\sim 1 \mathrm{~mm}^{3}\right)$, washed three times with PBS, then soaked in collagenase-I solution (Sigma-Aldrich) at $37^{\circ} \mathrm{C}$ and agitated for $60-90 \mathrm{~min}$. The digestion was terminated by Dulbecco's modified Eagle's medium (DMEM) containing 10\% serum. Subsequent to screening and washing with PBS, the stromal vascular fraction (SVF) cells were centrifuged at $400 \mathrm{x} \mathrm{g}$ for $10 \mathrm{~min}$ at room temperature. The DMEM/F12 containing $10 \%$ calf serum (Gibco-BRL) was used to culture the cells. The medium was replaced $12 \mathrm{~h}$ later in order to exclude the unattached cells and the culture medium was replaced every 2-3 days for the duration of the experiment. Following an additional 6-7 days, the diphenylmethane-4,4'-diisocyanate (MDI) induction method was initiated when the SVF cells exhibited contact inhibition. During the first 3 days of the MDI induction method the culture medium was DMEM/F12 containing fetal bovine serum (FBS) and 3-isobutyl-1-methylxanthine (IBMX; Sigma-Aldrich), then it was replaced by serum-free DMEM/F12 containing IBMX, dexamethasone (Dex) and insulin (In). The culture medium was replaced every 2-3 days until the appearance of lipid droplets.

Primary culture of murine BMDMs. Briefly, subsequent to anesthesia and immersion disinfection, the long bones of the mice were removed and soaked in conditioned culture medium (20\% L929 supernatants, 60\% serum-free DMEM, 20\% FBS, $2 \mathrm{mmol} / 1$ glutamine, $100 \mathrm{U} / \mathrm{ml}$ penicillin and $100 \mu \mathrm{g} / \mathrm{ml}$ streptomycin). The L929 supernatants were acquired as follows: $5 \times 10^{5}$ L929 cells were seeded into the $75 \mathrm{~cm}^{2}$ culture flasks and cultured in an incubator at $37^{\circ} \mathrm{C}, 5 \% \mathrm{CO}_{2}$ and saturated humidity for 7 days without medium change. Following harvesting, the supernatants were filtrated through the $0.22 \mu \mathrm{m}$ filter and then stored at $-70^{\circ} \mathrm{C}$. The two ends of the bones were cut off by eye scissors and marrow was eluted into $50 \mathrm{ml}$ conical tubes with conditioned culture medium using $10 \mathrm{ml}$ syringes and $25 \mathrm{G}$ needles. All products were purchased from Thermo Fisher Scientific (Pittsburgh, PA, USA) unless otherwise stated. The marrows were filtered through a 200-mesh screen (Thermo Fisher Scientific) to exclude impurities, such as adipose tissues. Subsequent to $10 \mathrm{~min}$ of centrifugation at $400 \mathrm{x} \mathrm{g}$ at room 
Table I. Primer sequences.

\begin{tabular}{lll}
\hline Gene & \multicolumn{1}{c}{ Forward primer (5'-3') } & \multicolumn{1}{c}{ Reverse primer $\left(5^{\prime}-3^{\prime}\right)$} \\
\hline$\beta$-actin & GGCTGTATTCCCCTCCATCG & CCAGTTGGTAACAATGCCATGT \\
IL-6 & TGGAGTCACAGAAGGAGTGGCTAAG & TCTGACCACAGTGAGGAATGTCCAC \\
IL-1 $\beta$ & CTGTGACTCGTGGGATGATG & GGGATTTTGTCGTTGCTTGT \\
Fas & GATCTGGGCTGTCCTGCCTCT & TTCACGAACCCGCCTCCTC \\
Fas-L & TGCAAGTGCAAACCAGACTTC & GTCAACAACCATAGGCGATTTCT \\
TGF- $\beta$ & CCGCAACAACGCCATCTATG & CTCTGCACGGGACAGCAAT \\
TNF- $\alpha$ & GTGACAAGCCTGTAGCCCA & AAAAGTAGACCTGCCCGGAC \\
MMP-2 & CAAGTTCCCCGGCGATGTC & TTCTGGTCAAGGTCACCTGTC \\
MMP-9 & CTGGACAGCCAGACACTAAAG & CTCGCGGCAAGTCTTCAGAG \\
COX-2 & TGCACTATGGTTACAAAAGCTGG & TCAGGAAGCTCCTTATTTCCCTT \\
MCP-1 & TTAAAAACCTGGATCGGAACCAA & GCATTAGCTTCAGATTTACGGGT
\end{tabular}

PAR-2, protease-activated receptor-2; IL, interleukin; MCP-1, monocyte chemoattractant protein-1; FasL, Fas ligand; TNF- $\alpha$, tumor necrosis factor- $\alpha$; TGF- $\beta$; transforming grow th factor- $\beta$; MMP, matrix metalloproteinase; COX-2, cyclooxygenase- 2 .

temperature, the cells were resuspended in the conditioned culture medium, seeded into a 6-well plate and incubated at $37^{\circ} \mathrm{C}$ in an atmosphere of $5 \% \mathrm{CO}_{2}$ and $95 \%$ humidity.

Subsequent to culture for an additional 3 days, the medium was replaced by DMEM, which contained $10 \%$ FBS. The medium was replaced every 2-3 days. Cells were used for experiments when the confluency approached $\sim 80 \%$.

Oil Red $O$ staining. Lipid droplets were observed by Oil Red $\mathrm{O}$ staining, which are a feature of mature adipocytes. Briefly, the cells were washed twice with PBS and fixed in $3.7 \%$ formaldehyde at room temperature for $1 \mathrm{~h}$, then washed three times with PBS and stained with $2 \mathrm{ml}$ oil red solution (Sigma-Aldrich) for a further $1 \mathrm{~h}$. Images were captured of the stained cells randomly under light microscopy.

Western blot analysis. BMDMs were lysed using radioimmunoprecipitation assay buffer lysis buffer (Beyotime Institute of Biotechnology, Shanghai, China) on ice for $30 \mathrm{~min}$, and then the cells were centrifuged at $4^{\circ} \mathrm{C}$ at $10,000 \times \mathrm{g}$ (Thermo Fisher Scientific) for $10 \mathrm{~min}$ to collect supernatants. A total of $30 \mu \mathrm{g}$ protein was separated by $10 \%$ SDS-PAGE (Merck, Kenilworth, NJ, USA) and transferred onto a polyvinylidene difluoride membrane (Invitrogen Life Technologies). Subsequent to blocking with $5 \%$ milk for $1 \mathrm{~h}$ at room temperature, the membrane was incubated with rabbit anti-mouse PAR-2 antibody (Abcam; cat. no. ab124227; 1:250) for $2 \mathrm{~h}$ and with the goat anti-rabbit IgG-horseradish peroxidase secondary antibody (Santa Cruz Biotechnology, Inc.; cat. no. sc-2054; 1:100) for $1 \mathrm{~h}$ at room temperature. The result was detected by enhanced chemiluminescence (Pierce, Biotechnology, Inc., Rockford, IL, USA) according to the manufacturer's instructions. GAPDH (Santa Cruz Biotechnology, Inc.; cat. no. sc-25778, 1:2,000) was used as a loading control.

Reverse transcription-quantitative polymerase chain reaction (RT-qPCR). RT-qPCR was conducted to quantify the expression levels of IL-1 $\beta$, IL-6, TNF- $\alpha$, cyclooxygenase (COX)-2,
MCP-1 and matrix metalloproteinase (MMP)-2 in the murine adipocytes with or without PAR-2 agonist peptide, SLIGKV (400 $\mu \mathrm{M}$; Sigma-Aldrich), as well as the expression levels of PAR-2, Fas, Fas-ligand (FasL), IL-1 $\beta$, IL-6, TNF- $\alpha$, MCP-1 and transforming growth factor (TGF)- $\beta$ in BMDMs. TRIzol reagent (Invitrogen Life Technologies) was used to extract the RNAs of the murine adipocytes and BMDMs. cDNAs were obtained using a reverse transcription kit (Toyobo, Osaka, Japan). The 20- $\mu$ l reaction mixture was comprised as follows: $5 \mu \mathrm{l}$ cDNA, $10 \mu \mathrm{l}$ SYBR Green Supermix (Bio-Rad Laboratories BV, Veenendaal, Netherlands), $1 \mu 1$ upstream and downstream primers $\left(0.5 \mu \mathrm{l}\right.$ each) and $4 \mu \mathrm{l} \mathrm{ddH_{2 }} \mathrm{O}$. The reaction conditions were as follows: $95^{\circ} \mathrm{C}$ Initial denaturation for $3 \mathrm{~min}$ ( $1 \mathrm{cycle}), 95^{\circ} \mathrm{C}$ denaturation for $15 \mathrm{sec}$, annealing for $30 \mathrm{sec}$ and $72^{\circ} \mathrm{C}$ extension for $15 \mathrm{sec}$ (40 cycles). Assays were carried out using the $\mathrm{iCycler}_{\mathrm{iQ}} \mathrm{T}^{\mathrm{TM}}$ Real-Time PCR detection system (Bio-Rad Laboratories, Inc., Hercules, CA, USA) with the primers shown in Table 1.

ELISA. ELISA was performed strictly following the manufacturer's instructions, in order to detect the expression levels of MCP-1 in the supernatants of the BMDMs and RAW264.7 cells. Optical density (OD) values at $450 \mathrm{~nm}$ were detected by the automatic microplate reader (GENios; Tecan Group Ltd., Männedorf, Switzerland).

Transwell cell migration assay. The Transwell cell migration assay was used to assess the migratory ability of RAW264.7 cells. The mature primary adipocytes were seeded into the lower chamber (density, $2 \times 10^{6} / \mathrm{ml}$ ) and the RAW264.7 were seeded into the upper chamber (volume, $200 \mu \mathrm{l} /$ well; cell density, 5x 10 $/ \mathrm{ml}$; Corning Incorporated, Corning, NY, USA). After a $24 \mathrm{~h}$ incubation at $37^{\circ} \mathrm{C}$ in a $5 \% \mathrm{CO}_{2}$ incubator (Thermo Fisher Scientific), the upper surface of the chamber filter was wiped off using a cotton swab. The cells that migrated through the filter were fixed and stained with $0.5 \%$ crystal violet (Sigma-Aldrich), and four fields were randomly selected for imaging and cell counting under an inverted microscope 
A

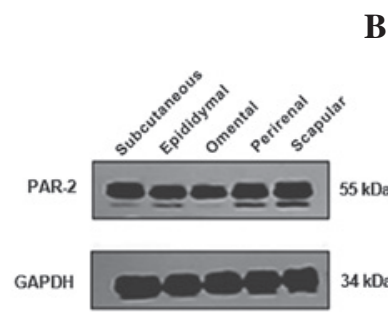

B

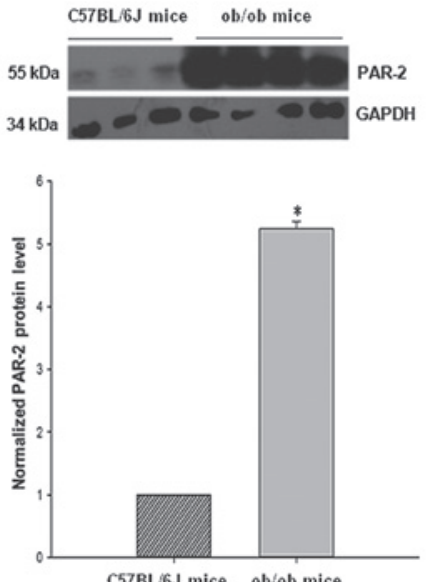

C
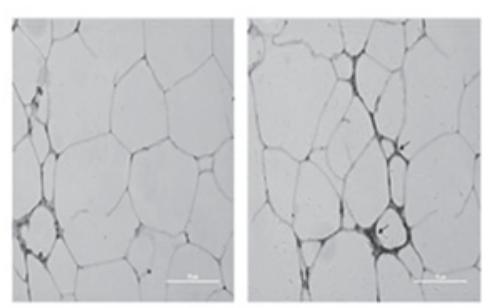

Figure 1. Macrophage infiltration and PAR-2 expression levels in the adipose tissues of mice. PAR-2 expression in different adipose tissue samples of (A) $\mathrm{C}_{57}$ mice and (B) $\mathrm{C}_{57}$ and ob/ob mice. ${ }^{*} \mathrm{P}<0.05$ vs. $\mathrm{C}_{57}$ mice; $\mathrm{n}=4$. (C) Macrophage infiltration in BKS-db/db mouse adipose tissue samples (magnification, $\mathrm{x} 100$ ). Left, negative control (BKS-db/db mouse epididymal adipose tissue treated by rabbit serum instead of F4/80 primary antibody); right, arrow indicates the specific marker (F4/80) of macrophages. A greater quantity of macrophages was observed in the adipose tissues of the obese mice when compared with those of the $\mathrm{C}_{57}$ mice. PAR-2, protease-activated receptor-2; $\mathrm{C}_{57}, \mathrm{C}_{57} \mathrm{BL} / 6 \mathrm{~J} ;$ ob/ob, B6.V-Lep $p^{\mathrm{ob}} / \mathrm{J} ; \mathrm{BKS}-\mathrm{db} / \mathrm{db}, \mathrm{BKS}(\mathrm{D})-$ Lepr $^{\mathrm{db} / \mathrm{J}}$.

A

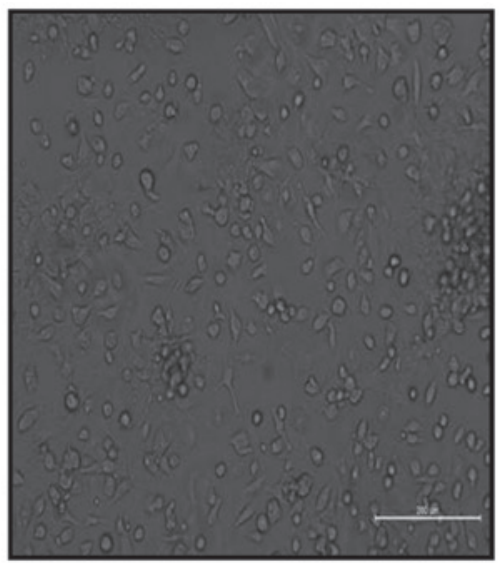

B

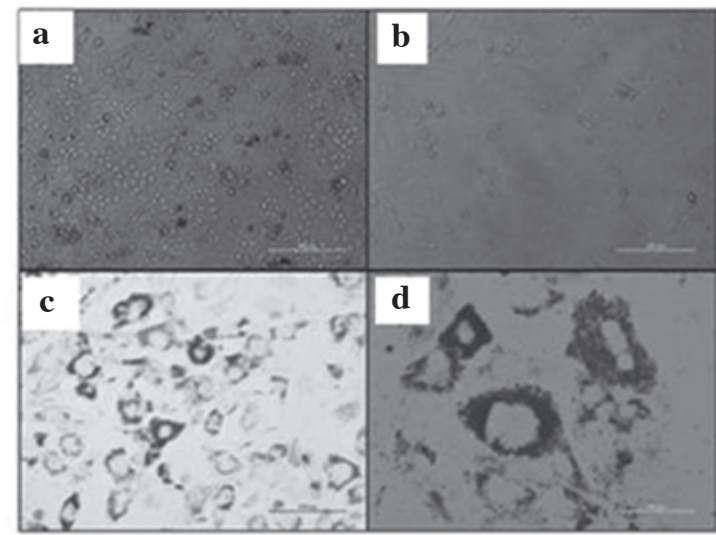

a
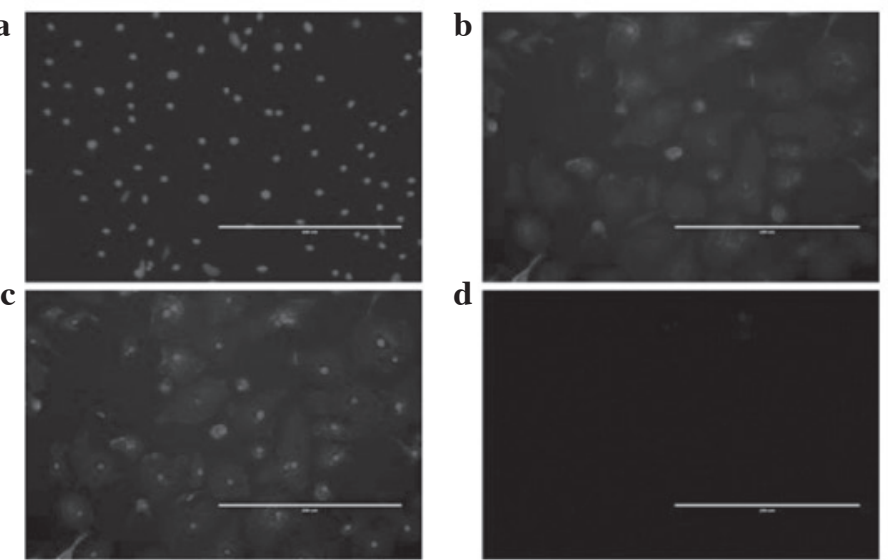

C

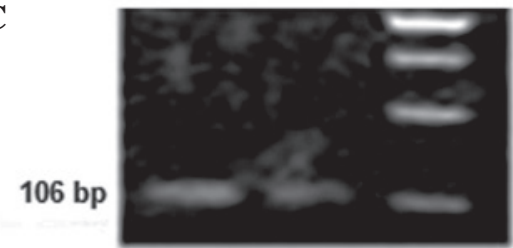

BMDM Adipocytes

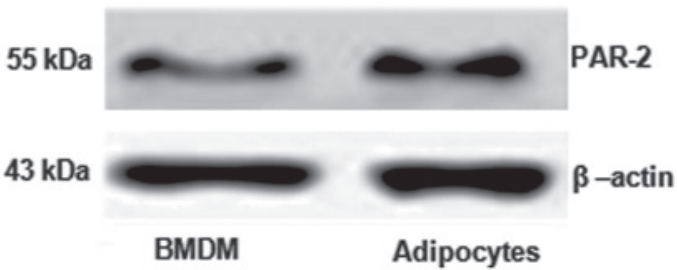

Figure 2. Expression of PAR-2 in murine adipocytes and BMDMs. (A) Culture and identification of BMDMs: Left, morphological features of BMDMs (day 9: High density of BMDMs; magnification, x100). Right, molecular markers of murine BMDMs (immunofluorescence); (a) DAPI staining (magnification, x400); (b) F4/80 (magnification, x400); (c) merged image (magnification, x400); (d) negative control (magnification, x400). (B) Morphological features of SVFs and adipocytes: (a) SVF (day 7; magnification, x200); (b) SVF (day 7; magnification, x200); (c) adipocytes (day 14: Oil Red O staining; magnification, x200); (d) adipocytes (day 14: Oil Red O staining; magnification, x200). (C) BMDMs and adipocytes express PAR-2 at an mRNA (upper, white bands) and protein (lower, black bands) level. PAR-2, protease-activated receptor-2; BMDM, bone marrow-derived macrophage; SVF, stromal vascular fraction.

(Olympus IX71; Olympus, Tokyo, Japan). Cells were lysed with $100 \mu 133 \%$ acetic acid solution (Sigma-Aldrich) in ethanol prior to reading the OD value for each well at $562 \mathrm{~nm}$ with the automatic microplate reader. 
Statistical analysis. The data are expressed as the mean \pm standard deviation and were analyzed using Student's t-test and one-way analysis of variance using SPSS software, version 13.0 (SPSS, Inc., Chicago, IL, USA). P $<0.05$ was considered to indicate a statistically significant difference.

\section{Results}

Macrophage infiltration and PAR-2 expression in the adipose tissues of mice. Western blotting was used to detect the PAR-2 expression levels in different adipose tissues in mice (Fig. 1A). PAR-2 expression was assessed in various adipose tissues of the $\mathrm{C}_{57}$ mice, including the subcutaneous, epididymal, scapular, perirenal and omental adipose tissues. The protein expression level of PAR-2 was observed to be significantly greater in the total adipose tissues of ob/ob mice when compared with that of the $\mathrm{C}_{57}$ mice (Fig. 1B; $\mathrm{P}<0.05$ ).

The presence of macrophages in the adipose tissues was observed by immunohistochemical staining of F4/80. Fig. 1C presents the F4/80-positive granules specific to the macrophages in the epididymal adipose tissues of BKS-db/db mice. Notably, a larger number of macrophages were observed in the adipose tissues of obese (BKS-db/db) mice than the $\mathrm{C}_{57}$ mice.

PAR-2 is expressed in the murine adipocytes and BMDMs. BMDMs and primary murine adipocytes were cultured and identified. As presented in Fig. 2A (left panel), following culture for 3 days, macrophage progenitor cells were observed, and 6-7 days later mature macrophages were observed by light microscopy. The BMDMs were digested and then placed upon the glass slides (in the 24-well plates). When the cells were reattached, immunohistochemical analysis of F4/80 was conducted. Figure 2A (right panel) illustrates the F4/80-positive granules in the BMDMs.

Fig. 2B presents the culture and identification of the primary murine adipocytes. Subsequent to the appearance of contact inhibition in the SVF cells (commonly at 6-7 days following cell attachment; Fig. 2Ba and $\mathrm{Bb}$ ), MDI induction was initiated: Culture for 3 days with DMEM/F12 (containing $10 \%$ calf serum and $11.5 \mathrm{mg} / \mathrm{ml}$ IBMX) then culture for 4-5 days with serum-free DMEM/F12 (containing $11.5 \mathrm{mg} / \mathrm{ml}$ IBMX, $1 \mathrm{mg} / \mathrm{ml} \mathrm{In} \mathrm{and} 390 \mathrm{ng} / \mathrm{ml}$ Dex). Lipid droplets were observed by Oil Red O staining (Fig. 2Bc and Bd), which are a feature of mature adipocytes.

RT-qPCR and western blotting of PAR-2 were performed subsequent to extracting the RNA and protein of the primary murine adipocytes and BMDMs (Fig. 2C; upper panel presents the mRNA expression and the lower panel presents the protein expression). As demonstrated in Fig. 2C, PAR-2 was observed to be expressed in the adipocytes and BMDMs.

PAR-2 agonists upregulate the mRNA expression of inflammation-associated cytokines in the murine primary adipocytes. Subsequent to a 12-h treatment with the PAR-2 agonist, the total RNAs of the adipocytes were extracted to detect the expression levels of the following inflammation-associated cytokines: IL-1 $\beta$, IL-6, TNF- $\alpha$, COX-2, MCP-1 and MMP-2. The results of RT-qPCR indicated that the mRNA expression levels of IL-1 $\beta$, IL-6, TNF- $\alpha$ and COX-2 were significantly

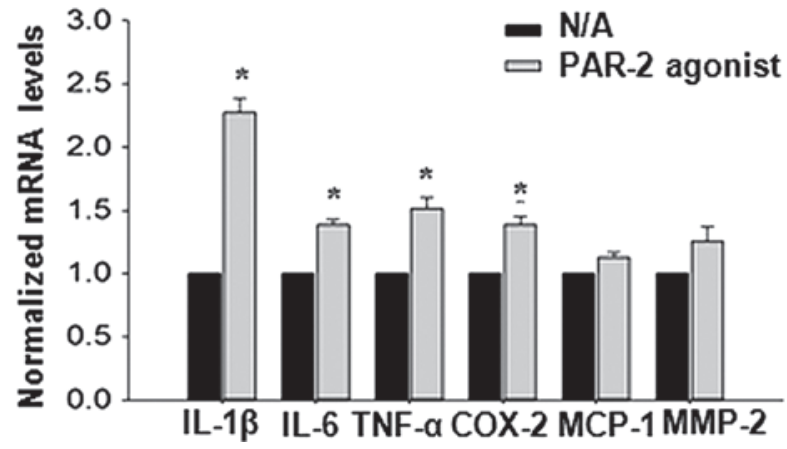

Figure 3. Expression levels of inflammatory cytokines, IL-1 $\beta$, IL-6, TNF- $\alpha$, COX-2, MCP-1 and MMP-2 were detected in adipocytes by reverse transcription-quantitative polymerase chain reaction. Among the six cytokines, the expression levels of IL-1 $\beta$, IL-6, TNF- $\alpha$ and COX-2 were significantly increased by the PAR-2 agonist. ${ }^{*} \mathrm{P}<0.05$ vs. the normal control group; $\mathrm{n}=4$. IL, interleukin; TNF- $\alpha$, tumor necrosis factor- $\alpha$; COX-2, cyclooxygenase-2; MCP-1, monocyte chemoattractant protein-1; MMP-2, matrix metalloproteinase-2; PAR-2, protease-activated receptor-2.

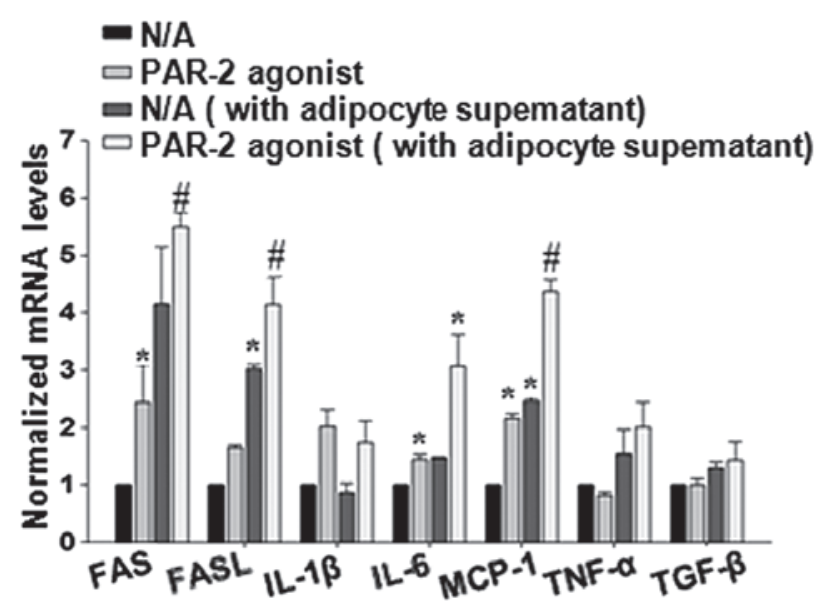

Figure 4. Adipocytes and PAR-2 agonists promote mRNA expression levels of inflammatory cytokines in BMDMs. Expression levels of Fas, IL-6 and MCP-1 were significantly higher in the PAR-2 agonist and the adipocyte supernatants group than in the control group, respectively. Additionally, compared with the adipocyte supernatant group, the co-culture of adipocyte supernatants and PAR-2 agonists further increased the mRNA expression levels of Fas, FasL and MCP-1. ${ }^{*} \mathrm{P}<0.05$ vs. the N/A group; $n=4$. ${ }^{\#} \mathrm{P}<0.01$ vs. the N/A group; $\mathrm{n}=4$. PAR-2, protease-activated receptor-2; IL, interleukin; MCP-1, monocyte chemoattractant protein-1; FasL, Fas ligand; TNF- $\alpha$, tumor necrosis factor- $\alpha$; TGF- $\beta$; transforming growth factor- $\beta$.

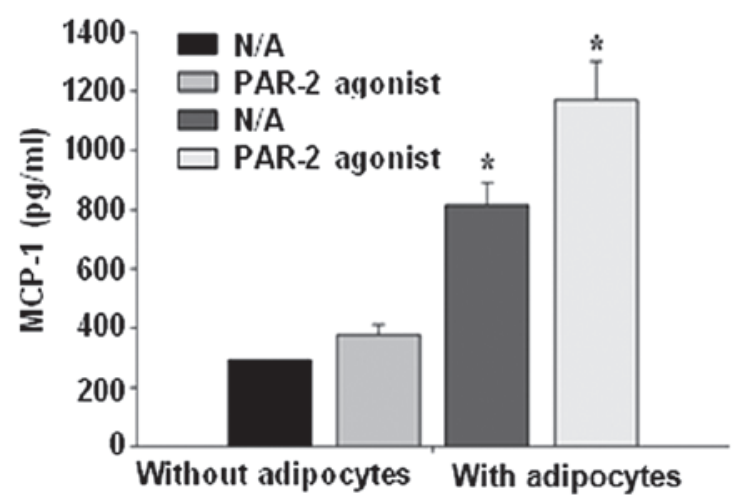

Figure 5. ELISA of MCP-1 in BMDMs. Adipocytes and the PAR-2 agonist promote the expression levels of MCP-1 in BMDMs. ${ }^{*} \mathrm{P}<0.05$ vs. the N/A group; $\mathrm{n}=4$. MCP-1, monocyte chemoattractant protein-1; BMDMs, bone marrow-derived macrophages; PAR-2, protease-activated receptor-2. 
A

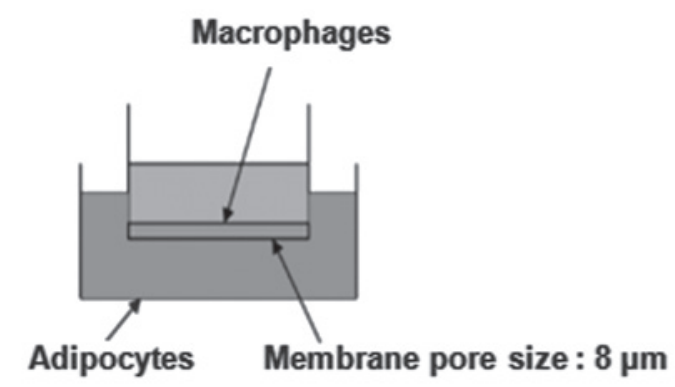

B

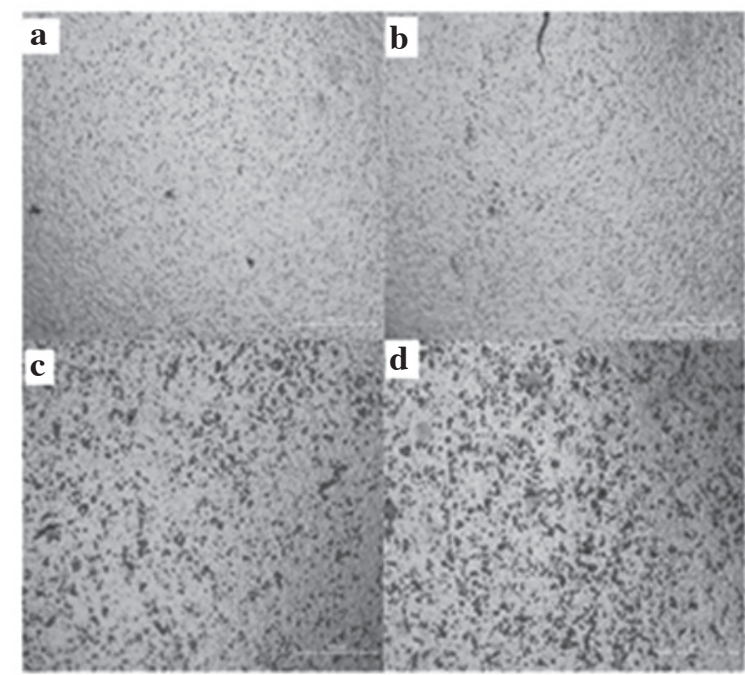

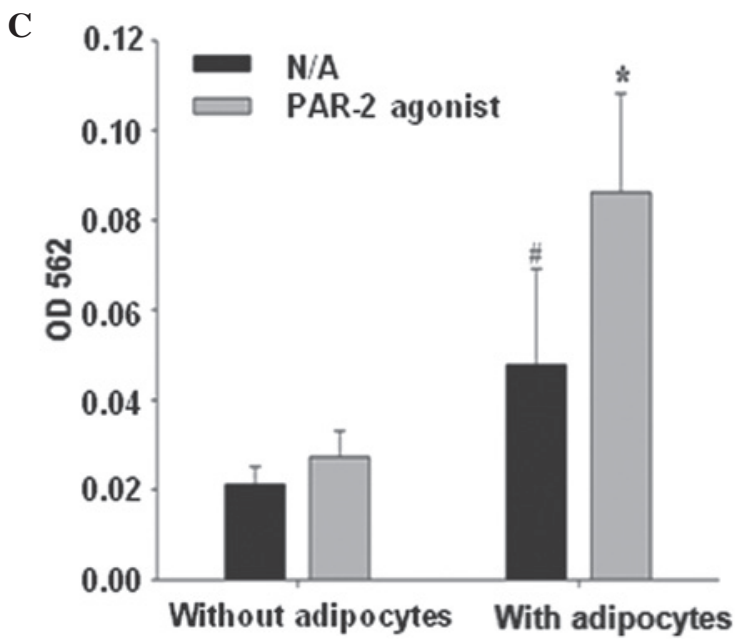

Figure 6. Effect of adipocytes and the PAR-2 agonist on the migratory ability of RAW264.7 cells. (A) Schematic diagram of the Transwell cell migration assay. (B) Adipocytes and the PAR-2 agonist promote the migratory ability of RAW264.7 cells. (a) RAW264.7 cells cultured without the PAR-2 agonist and adipocyte supernatants; (b) RAW264.7 cells cultured without adipocyte supernatants, but with the PAR-2 agonist; (c) RAW264.7 cultured with adipocyte supernatants, but without the PAR-2 agonist; (d) RAW264.7 cultured with adipocyte supernatants and the PAR-2 agonist. (C) OD values of crystal violet in the different treatment groups. ${ }^{\prime} \mathrm{P}<0.05$ vs. the N/A group $(\mathrm{n}=4) ;{ }^{*} \mathrm{P}<0.05$ vs. the adipocytes group or the PAR-2 agonist group ( $\left.\mathrm{n}=4\right)$. PAR-2, protease-activated receptor-2; OD, optical density.

higher in the treatment group when compared with the control group (Fig. 3; $\mathrm{P}<0.05$ ).

Adipocytes and PAR-2 agonists upregulate mRNA expression levels of inflammation-associated cytokines in the BMDMs. Subsequent to treatment with the supernatants of the adipocytes and PAR-2 agonists, simultaneously or separately, the total RNAs of the BMDMs were extracted to detect the expression levels of the following inflammation-associated cytokines: Fas, FasL, IL-1 $\beta$, IL- 6 , TNF- $\alpha$, MCP- 1 and TGF- $\beta$. The results indicated that the mRNA expression levels of Fas, IL-6 and MCP-1 were significantly increased in the PAR-2 agonist group when compared with the control group $(\mathrm{P}<0.05)$. In addition, mRNA expression levels of FasL, IL- 6 and MCP-1 were also significantly higher in the adipocyte supernatant group than in the control group $(\mathrm{P}<0.05)$. Additionally, it was observed that compared with the adipocyte supernatant group, the co-culture of adipocyte supernatants and PAR-2 agonists further increased the mRNA expression levels of Fas, FasL and MCP-1 (Fig. 4; P<0.01).
Adipocytes and PAR-2 agonists upregulate protein expression levels of MCP-1 in the BMDMs. Subsequent to treatment with the supernatants of the adipocytes and PAR-2 agonists simultaneously or separately, the expression levels of MCP-1 in BMDMs were measured using the ELISA assay. The results indicated that the adipocytes and PAR-2 agonists upregulated the expression levels of MCP-1 in BMDMs (Fig. 5).

Adipocytes and PAR-2 agonists promote the migratory ability of RAW264.7 cells. The cell distribution is presented in Fig. 6A. RAW264.7 cells at a concentration of $5 \times 10^{5}$ cells/well were used in the current study. PAR-2 agonists were evenly added into the serum-free DMEM used in the lower chambers with or without adipocytes. Cell migrations were measured subsequent to a 12 -h co-incubation. Fig. $6 \mathrm{~B}$ presents the microscopic view of the migration and Fig. 6C displays the OD values of crystal violet dissolved in the $33 \%$ acetic acid. It was identified that PAR-2 agonists and adipocytes significantly promoted the migratory ability of RAW264.7 cells separately and synergistically $(\mathrm{P}<0.05$ vs. N/A). In addition, compared 
with either of the single treatment groups, co-incubation of PAR-2 agonists and adipocytes further enhanced the migratory ability of RAW264.7 cells $(\mathrm{P}<0.05)$.

\section{Discussion}

Chronic low-grade systemic inflammation is one of the features and fundamental pathological processes in obesity-associated diseases (13). As an important endocrine organ, adipose tissue is able to secrete numerous hormones and cytokines, including IL-6, TNF- $\alpha$, IL-8, IL-1 $\beta$, MCP-1, leptin and adiponectin (14). Previous studies have demonstrated that chronic low-grade systemic inflammation is closely associated with endocrine dysfunction in adipose tissues (15). Cottam et al (4) demonstrated that the serous levels and adipose concentrations of IL-6 and TNF- $\alpha$ were significantly increased in obese patients (4), while IL- 6 and TNF- $\alpha$ may interfere with the normal differentiation of adipocytes, and induce their differentiation into pro-inflammatory macrophages (16). Adipocyte-secreted adiponectin has been previously identified to reduce the TNF- $\alpha$ expression in adipose tissues, however also reduces macrophage infiltration into the adipose tissues (17). Therefore, adipocyte-secreted inflammatory factors have been suggested to participate and serve an important role in the pathogenesis of obesity.

In recent years, the observation of significant infiltration of inflammatory cells within adipose tissue samples has become a cause of concern; it was previously reported that a greater number of adipose macrophages were observed in obese mice and humans compared with normal adipose tissues $(18,19)$, which is consist with the results of the current study. Further studies have demonstrated that obesity is associated with macrophage polarization towards the M1 type of pro-inflammatory macrophages (20) and M1 macrophages may secrete inflammation-associated cytokines, including IL-6, TNF- $\alpha$ and IL-12 (21). Therefore, the obese adipose tissue is hypothesized to be a significant source of pro-inflammatory factors in the circulation. Thus, the current study detected the expression levels of multiple inflammation-associated cytokines in the BMDMs of different treatment groups.

Increasing evidence indicates that mast cells are critical for the pathogenesis of inflammatory diseases (22). Proteases released from mast cells have been reported to stimulate PARs, induce microleakage and result in widespread inflammation (23). With serine kinase as the specific ligand, PAR-2 is the only member in the PAR family that is insensitive to thrombin (24). PAR-2 has been identified to have extensive effects on inflammatory responses in various organs and tissues. Lewkowich et al (25) suggested that activation of PAR-2 in the myeloid dendritic cells may result in allergic airway inflammations. Lindner et al (26) activated PAR-2 in the vascular endothelial cells, demonstrating that this leads to rapid P-selectin-induced leukocyte rolling; however, this phenomenon was observed to be absent in PAR2-deficient mice, indicating that there was a delayed onset of inflammation in PAR-2-deficient mice. Previous studies demonstrated that PAR-2 activation in the vascular endothelial cells significantly increased the recruitment of monocyte macrophages and promoted their capacity to secrete IL-6, TNF- $\alpha$ and IL-1 $\beta$ (27).
The results of the present study demonstrate that PAR-2 was expressed in the adipose tissues of $\mathrm{C}_{57}$ mice and obese mice (db/db and ob/ob) and the PAR-2 protein expression level was significantly higher in the adipose tissues of the ob/ob mice than in the $\mathrm{C}_{57}$ mice $(\mathrm{P}<0.05)$. This indicates that PAR-2 may have pro-inflammatory effects in the obese adipose tissues. It was observed that PAR-2 activation significantly increased mRNA expression levels of inflammation-associated cytokines in the murine primary adipocytes, which was consistent with the results of Badeanlou et al (28). Subsequently, the Transwell cell migration assay was conducted to assess the migratory ability of the RAW264.7 murine macrophage cell lines and it was observed that PAR-2 agonists and adipocytes significantly promoted the migratory ability of RAW264.7 cells $(\mathrm{P}<0.05)$. Furthermore, compared with either treatment alone, co-incubation of PAR-2 agonists with adipocytes further enhanced the migratory ability of RAW264.7 cells $(\mathrm{P}<0.05)$. Additionally, it was identified with the co-incubation of BMDMs and murine primary adipocytes that PAR-2 activation significantly promoted the mRNA expression levels of Fas, FasL, IL-1 $\beta$, IL-6, MCP-1, TNF- $\alpha$ and TGF- $\beta$ in BMDMs (all P<0.05). MCP-1 has been reported to be involved in the recruitment of macrophages (29) and in the results from the ELISA in the current study, PAR-2 activation significantly increased the secretion of MCP-1 in BMDMs $(\mathrm{P}<0.05)$. Therefore this may be the cause of the increased infiltration of macrophages identified in the adipose tissues.

In conclusion, it is proposed that PAR-2 participates in the chronic low-grade inflammatory process observed in obesity, and inhibition of PAR-2 activation may be a potential novel therapeutic target for obesity-associated diseases.

\section{Acknowledgements}

The current study was funded by the National Natural Science Foundation of China (grant nos. 81001170, 81172507 and 81270490), the Natural Science Foundation of Ningxia (grant no. nz14074) and by the Scientific Research Ability Training and Enhancement Project of Basic Medical Science Platform of Fudan University (grant no. J1210041).

\section{References}

1. Trayhurn P: Endocrine and signalling role of adipose tissue: New perspectives on fat. Acta Physiol Scand 184: 285-293, 2005.

2. Lasselin J and Capuron L: Chronic low-grade inflammation in metabolic disorders: Relevance for behavioral symptoms. Neuroimmunomodulation 21: 95-101, 2014.

3. Martinez-Santibañez G and Lumeng CN: Macrophages and the regulation of adipose tissue remodeling. Annu Rev Nutr 34: 57-76, 2014

4. Cottam DR, Mattar SG, Barinas-Mitchell E, Eid G, Kuller L, Kelley DE and Schauer PR: The chronic inflammatory hypothesis for the morbidity associated with morbid obesity: Implications and effects of weight loss. Obes Surg 14: 589-600, 2004.

5. Altintas MM, Rossetti MA, Nayer B, Puig A, Zagallo P, Ortega LM, Johnson KB, McNamara G, Reiser J, Mendez AJ and Nayer A: Apoptosis, mastocytosis and diminished adipocytokine gene expression accompany reduced epididymal fat mass in long-standing diet-induced obese mice. Lipids Health Dis 10: 198, 2011

6. Liu J, Divoux A, Sun J, et al: Genetic deficiency and pharmacological stabilization of mast cells reduce diet-induced obesity and diabetes in mice. Nat Med 15: 940-945, 2009. 
7. Lim J, Iyer A, Liu L, Suen JY, Lohman RJ, Seow V, Yau MK, Brown L and Fairlie DP: Diet-induced obesity, adipose inflammation, and metabolic dysfunction correlating with PAR2 expression are attenuated by PAR2 antagonism. FASEB J 27: 4757-4767, 2013.

8. Rothmeier AS and Ruf W: Protease-activated receptor 2 signaling in inflammation. Semin Immunopathol 34: 133-149, 2012.

9. Lu J, Chen B, Li S and Sun Q: Tryptase inhibitor APC 366 prevents hepatic fibrosis by inhibiting collagen synthesis induced by tryptase/protease-activated receptor 2 interactions in hepatic stellate cells. Int Immunopharmacol 20: 352-357, 2014.

10. Lu C,Zhao FD, Li XB and Yin LH: Up regulation of interleukin-8 expressions induced by mast cell tryptase via protease activated receptor-2 in endothelial cell line. Chin Med J (Engl) 118: 1900-1906, 2005.

11. Ma Y, Zhang B, Qian R, Lu C, Zhao F and Yin L: Tryptase activates $P K B$ in inflammatory reaction in ECV304 cells. Biochim Biophys Acta 1763: 313-321, 2006.

12. Jiang YF, Zhao FD, Li XB, Ning YX, Zhi XL, Qian RZ and Yin LH: Effects of RNA interference-induced tryptase down-regulation in P815 cells on IL-6 and TNF-alpha release of endothelial cells. J Zhejiang Univ Sci B 9: 656-661, 2008.

13. Ilich JZ, Kelly OJ, Kim Y and Spicer MT: Low-grade chronic inflammation perpetuated by modern diet as a promoter of obesity and osteoporosis. Arh Hig Rada Toksikol 65: 139-148, 2014.

14. Adamczak M and Wiecek A: The adipose tissue as an endocrine organ. Semin Nephrol 33: 2-13, 2013.

15. Ouchi N, Parker JL, Lugus JJ and Walsh K: Adipokines in inflammation and metabolic disease. Nat Rev Immunol 11: 85-97, 2011.

16. Gustafson B and Smith U: Cytokines promote Wnt signaling and inflammation and impair the normal differentiation and lipid accumulation in 3T3-L1 preadipocytes. J Biol Chem 281: 9507-9516, 2006.

17. Kim JY, van de Wall E, Laplante M, Azzara A, Trujillo ME, Hofmann SM, Schraw T, Durand JL, Li H, Li G, et al: Obesity-associated improvements in metabolic profile through expansion of adipose tissue. J Clin Invest 117: 2621-2637, 2007.

18. Weisberg SP, McCann D, Desai M, Rosenbaum M, Leibel RL and Ferrante AW Jr: Obesity is associated with macrophage accumulation in adipose tissue. J Clin Invest 112: 1796-1808, 2003.
19. Xu H, Barnes GT, Yang Q, Tan G, Yang D, Chou CJ, Sole J, Nichols A, Ross JS, Tartaglia LA and Chen H: Chronic inflammation in fat plays a crucial role in the development of obesity-related insulin resistance. J Clin Invest 112: 1821-1830, 2003.

20. Lumeng CN, Bodzin JL and Saltiel AR: Obesity induces a phenotypic switch in adipose tissue macrophage polarization. J Clin Invest 117: 175-184, 2007.

21. Mantovani A, Sica A, Sozzani S, Allavena P, Vecchi A and Locati M: The chemokine system in diverse forms of macrophage activation and polarization. Trends Immunol 25: 677-686, 2004.

22. Theoharides TC, Alysandratos KD, Angelidou A, Delivanis DA, Sismanopoulos N, Zhang B, Asadi S, Vasiadi M, Weng Z, Miniati A and Kalogeromitros D: Mast cells and inflammation. Biochim Biophys Acta 1822: 21-33, 2012.

23. Schmidlin F and Bunnett NW: Protease-activated receptors: How proteases signal to cells. Curr Opin Pharmacol 1: 575-582, 2001.

24. Ossovskaya VS and Bunnett NW: Protease-activated receptors: Contribution to physiology and disease. Physiol Rev 84: 579-621, 2004.

25. Lewkowich IP, Day SB, Ledford JR, Zhou P, Dienger K, Wills-Karp $M$ and Page K: Protease-activated receptor 2 activation of myeloid dendritic cells regulates allergic airway inflammation. Respir Res 12: 122, 2011

26. Lindner JR, Kahn ML, Coughlin SR, Sambrano GR, Schauble E, Bernstein D, Foy D, Hafezi-Moghadam A and Ley K: Delayed onset of inflammation in protease-activated receptor-2-deficient mice. J Immunol 165: 6504-6510, 2000.

27. Mamatzunun M, Ma YJ, Lu C, Zhao FD and Yin LH: Effect of tryptase on the expression of IL- 6 and TNF- $\alpha$ in microvascular endothelial cell. Fudan Univ J Med Sci 33: 217-221, 2006.

28. Badeanlou L, Furlan-Freguia C, Yang G, Ruf W and Samad F: Tissue factor-protease-activated receptor 2 signaling promotes diet-induced obesity and adipose inflammation. Nat Med 17: 1490-1497, 2011.

29. Kanda H, Tateya S, Tamori Y, Kotani K, Hiasa K, Kitazawa R, Kitazawa S, Miyachi H, Maeda S, Egashira K and Kasuga M: MCP-1 contributes to macrophage infiltration into adipose tissue, insulin resistance and hepatic steatosis in obesity. J Clin Invest 116: 1494-1505, 2006. 